\title{
Neuro-fuzzy approach to real-time transient stability prediction based on synchronized phasor measurements
}

\author{
Chih-Wen Liu ${ }^{\mathrm{a}, *}$, Shuenn-Shing Tsay ${ }^{\mathrm{a}}$, Yi-Jen Wang ${ }^{\mathrm{a}}$, Mu-Chun Su ${ }^{\mathrm{b}}$ \\ a Department of Electrical Engineering, National Taiwan University, Taipei, Taiwan, ROC \\ ${ }^{\mathrm{b}}$ Department of Electrical Engineering, Tamkang University, Taipei, Taiwan, ROC \\ Received 18 May 1998; received in revised form 5 June 1998; accepted 5 June 1998
}

\begin{abstract}
With new systems capable of making synchronized phasor measurements there are possibilities for real-time assessment of the stability of a transient swing in power systems. In the future, on-line control will be necessary as operating points are pushed closer toward the margin and fast reaction time becomes critical to the survival of the system. In this paper we develop a novel class of fuzzy hyperrectangular composite neural networks which utilize real-time phasor angle measurements to provide fast transient stability prediction for use with high-speed control. From simulation tests on a sample power system, it reveals that the proposed tool can yield a highly successful prediction rate in real-time. (C) 1999 Elsevier Science S.A. All rights reserved.
\end{abstract}

Keywords: Phasor measurement unit (PMU); Real-time transient stability prediction; Fuzzy hyperrectangular composite neural network (FHRCNN)

\section{Introduction}

With the advent of phasor measurement units (PMUs) [1-3] capable of tracking the dynamics of an electric power system, and with modern telecommunication abilities, utilities are becoming able to respond intelligently to an event in progress. By synchronizing sampling of microprocessor-based systems, phasor calculations can be placed on a common reference [3]. The magnitudes and angles of these phasors comprise the state of the power system and are used in state estimation and transient stability analysis. By communicating time-tagged phasor measurements to a central location the dynamic state of the system can be tracked in real-time. An emerging application of this technology is to track the state of the system immediately following a transient event to select an appropriate remedial control action. One such real-time control strategy is already being implemented at the Florida-Georgia interface [4], and others are currently under development [5]. More specifically, use can be made of these measurements to predict a developing swing and instantaneously initiate important relay operations such as

\footnotetext{
* Corresponding author. E-mail: cwliu@cc.ee.ntu.edu.tw
}

out-of-step blocking and tripping, or other control actions such as fast-value control of turbines [6], dynamic braking, superconducting magnetic energy storage system [7], system switching, or modulation of high voltage direct current (HVDC) link power flow [8].

There are obvious differences between the real-time stability prediction problem and off-line stability assessments. In conventional off-line transient stability assessments [12-14], in which the critical clearing time (CCT) is to be found, there are three stages that a power system goes through: prefault, fault-on and postfault stages. In the prediction problem, the CCT is not of interest. Instead, one can monitor the progress of the transient in real-time, thanks to the technique of synchronized phasor measurements. Moreover, we assume in a real-time problem that protection systems for faulted transmission lines are extremely fast and the fault is removed immediately at the fault inception. By ignoring the short fault-on stage (in which the transient phasor measurements are discarded) in real-time, the prediction problem only involves prefault and postfault stages.

On the other hand, many existing transient stability assessment techniques, while simple in off-line application are for real-time use. What is required is a compu- 
tationally efficient way of processing the real-time measurements to determine whether an evolving swing will ultimately be stable or unstable $[9,15]$ the possibility of using the short-term prediction algorithm for this propose was explored. In the paper a novel two-layer fuzzy hyperrectangular composite neural network (FHRCNN) is presented for real-time transient stability prediction using synchronized phasor measurements. This neuro-fuzzy approach can learn in off-line from training sets and are used in on-line to predict future behaviour of new data much faster than would be possible by solving the model analytically. First the technique of measuring synchronized phasor is introduced. Then we present a novel class of FHRCNN for prediction use. Finally, simulation results are shown to demonstrate the validity of the proposed method.

\section{The proposed neuro-fuzzy approach}

In a real-time environment it is desirable to predict the stability of transient swings before asynchronism occurs, then appropriate control actions such as tripping of generators or tie-lines can be initiated to protect system equipment or an identification of recoverable swings in order to avoid unnecessary tripping to maintain the integrity of the system. That would require that computations be carried out and decisions be made in very short periods of time. This is beyond the computational capability of current transient stability analysis methods.

Therefore a novel two-layer fuzzy hyperrectangular composite neural network (FHRCNN) is proposed for real-time stability prediction. The FHRCNN is a type of classifier that can be constructed off-line from a training set of examples. The FHRCNN is used to classify a transient swing as either stable or unstable on the basis of synchronized phasor measurements. Rather than attempting to solve the power system model in real-time, extensive simulations are carried out off-line in order to capture the essential features of system behaviour. The network building process statistically analyzes this data and constructs a FHRCNN designed to correctly classify new, unseen examples. The resulting FHRCNN classifier is compact and extremely fast, thus well suited for on-line use.

A description of basic elements of two-layer FHRCNN (Fig. 1) follows.

\subsection{Input vector (the predictor)}

$x=\left[x_{1}, x_{2}, \ldots, x_{n}\right]$ Is an input vector for FHRCNN and is also the predictor for real-time transient stability prediction task. In this paper the predictor $x$ is made by the following process.

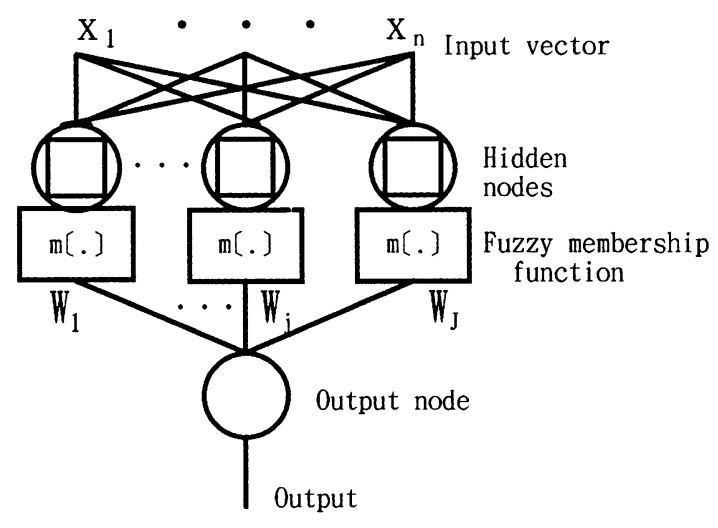

Fig. 1. A two-layer FHRCNN.

Stability prediction is based on an eight cycle window of phasor measurements which begins at fault clearing time, $T_{\mathrm{c}}$. Three consecutive measurements, four cycles apart, are taken from each of the total generator angles: The first measurement at $T_{\mathrm{c}}$, another at $T_{\mathrm{c}}+4 / 60$, and the last at $T_{\mathrm{c}}+8 / 60$ (Fig. 2). The generator angles, measured in radians and in center of inertia coordinates (COI), are first written to a data file in a format with three digits after the decimal.

Two velocities and one acceleration are computed from generator angles, for a total of six predictors per generator. Denoting the three angle measurements from the $i$ th generator $\delta_{i}(0), \delta_{i}(1), \delta_{i}(2)$, we compute

$V_{i}(0)=10^{*}\left[\delta_{i}(1)-\delta_{\mathrm{i}}(0)\right]$
$V_{i}(1)=10^{*}\left[\delta_{i}(2)-\delta_{i}(1)\right]$
$a_{i}(0)=20^{*}\left[\delta_{i}(2)-2 * \delta_{i}(1)+\delta_{i}(0)\right]$.

Thus the predictor, $x$, is of the following form:

$$
\begin{aligned}
x= & {\left[\delta_{1}(0), \delta_{1}(1), \delta_{1}(2), V_{1}(0), V_{1}(1), a_{n}(0), \ldots, \delta_{n}(0),\right.} \\
& \left.\delta_{n}(1), \delta_{n}(2), V_{n}(0), V_{n}(1), a_{n}(0)\right] .
\end{aligned}
$$

Remark 1: although PMU provides terminal bus phasors, it is possible to compute generator (rotor)

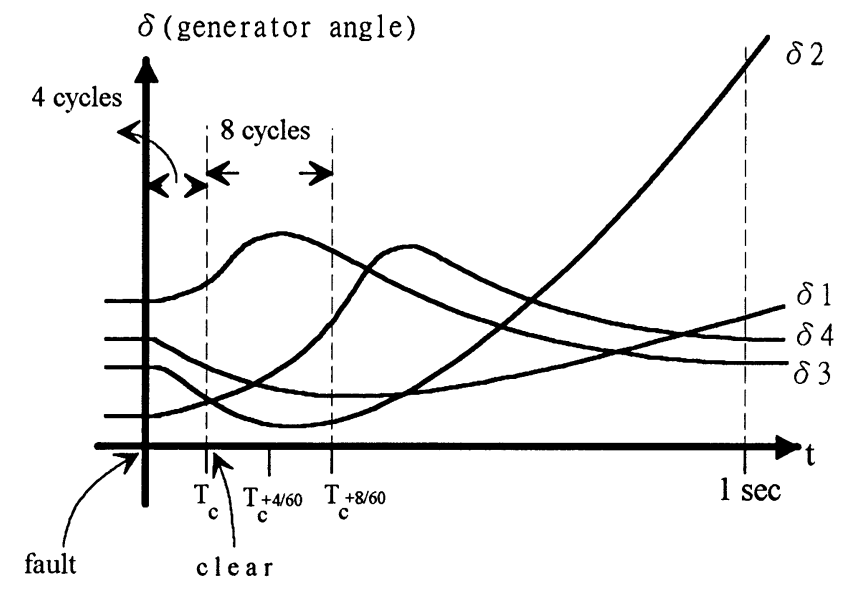

Fig. 2. Reading of generator angles. 
angles from terminal bus angles by mean of network admittance equation.

Remark 2: depending on the swings to be predicted, local devices compute bus voltage angles or generator angles from terminal bus angles, then transmit these angles to central center where transient stability predictions are conducted. Although synchronized phasor measurements are carried out constantly at a high sampling rate, say, $12 \times$ per cycle [3], we should consider the time for the transmission of angle data. It is reasonable to assume that we can obtain all synchronized data of interest at a rate of once every four cycles.

\subsection{Output variable}

The magnitude of output variable is employed to label stability status of transient swing. We use two integers to label the status like the following:

$1 \rightarrow$ unstable swing

$0 \rightarrow$ stable swing.

\subsection{I-O mapping}

The relationships between input vector and output variable of FHRCNN can be described by the following equation.

output $=\sum_{j+1}^{j} w_{j} m_{j}(\hat{x})+\theta$.

Where

$m_{j}(\hat{x})=\frac{\operatorname{vol}_{j}}{\operatorname{vol}_{j}+s_{j}^{2}\left(\operatorname{vol}_{j}-\operatorname{vol}_{j}\right)}$

is the fuzzy membership function,

$\operatorname{vol}_{j}=\prod_{i=1}^{n}\left(M_{j i}=m_{j i}\right)$

is the volume of the $j$ th hyperrectangular hidden node, and

$\operatorname{vol}_{j}(\hat{x})=\prod_{i=1}^{n} \max \left(M_{j i}-m_{j i}, x_{i}-m_{j i}, M_{j i}-x_{i}\right)$,

where $w_{j}, \theta, s_{j}, M_{j i}, m_{j i}$ are the scalar parameters adjusted by hybrid training algorithm.

\subsection{Hybrid training algorithm}

The supervised decision-directed learning (SDDL) algorithm and back-propagation (BP) algorithm are combined to train FHRCNN. The SDDL is based on an approach that divides the input vector space into proper subsets (hyperrectangles). Each hyperrectangle, corresponding to a hidden node, is $n$-dimensional and defined by $\left[m_{j 1}, M_{j 1}\right] \times\left[m_{j n}, M_{j n}\right]$. Once the number of hyperrectangles and initial parameters, $m_{j 1}, M_{j 1}, m_{j n}$,

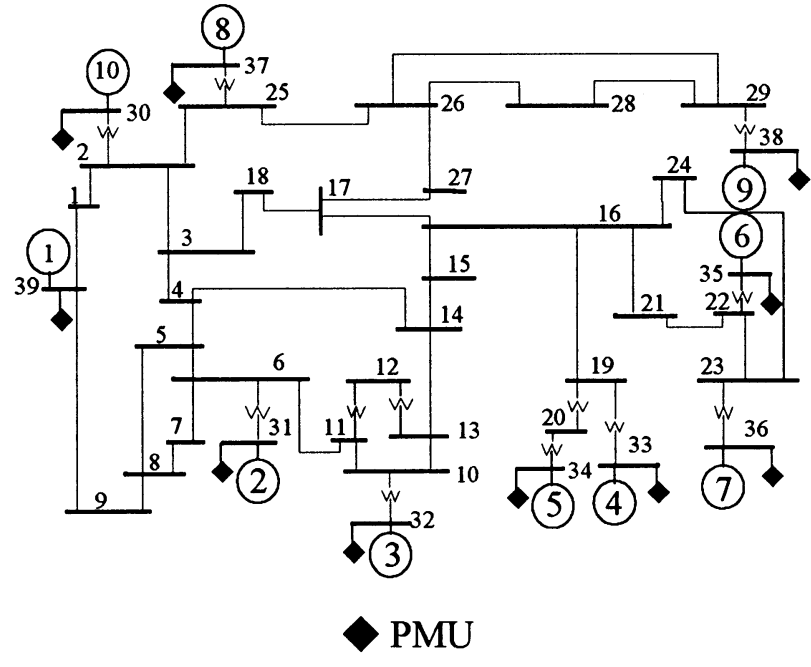

Fig. 3. One-line diagram of IEEE 39-bus system equipped by 10 PMUs.

$M_{j n}$, are determined by SDDL. The BP algorithm is used to adjust parameters, $w_{j}, \theta, s_{j}, M_{j i}, m_{j i}$, such that the following error function is minimized.

Error $=\sum_{p} E_{p}=\sum_{p} 1 / 2\left(\mathrm{Out}_{p}-t_{p}\right)^{2}$

where $t_{p}$ is the desired output value of the $p$-th vector, and $\mathrm{Out}_{p}$ is the output value from FHRCNN.

For the sake of brevity, a detailed description of the hybrid training algorithm is provided in [10].

\section{Simulation results}

The FHRCNN was investigated using the IEEE 39bus test system as shown in Fig. 3. In our simulation model, the generator is modeled by seventh-order differential equations and the loads are modeled as constant impedances. A detailed description of the above model can be referred to in Ref. [11]. Three-phase short-circuit-to-ground faults with four-cycle clearing time are simulated to occur on various transmission lines. The postfault system configuration is the same as the prefault system except that the faulted line is removed. Each example of a fault contains the simulated postfault phasor measurements along with whether the particular fault results in instability. Large numbers of examples are aggregated together into training and test sets, from which FHRCNN are constructed and tested.

\subsection{Instability criterion}

The criterion for instability is whether the difference between any two generator angles exceeds $\pi$ radian in the first second after clearing time. Otherwise the fault is declared as stable. We would like to emphasize that 
Table 1

Classification rates of FHRCNN and FNN for stability prediction

\begin{tabular}{lllll}
\hline Network & \multicolumn{2}{l}{ Training set $(\%)$} & \multicolumn{2}{l}{ Test set $(\%)$} \\
\hline Type & Stable & Unstable & Stable & Unstable \\
FHRCNN & 99.8 & 99.5 & 95.1 & 94.2 \\
FNN & 73.5 & 73.3 & 72.7 & 72.4 \\
\hline
\end{tabular}

the first second after clearing time. Otherwise the fault is declared as stable. We would like to emphasize that the instability criterion depends heavily on the characteristics of specific power systems. Here, the chosen $\pi$ radian is just for illustration of our scheme.

\subsection{Training sets and test sets}

For a given fault location, duration and clearing action, the fault-on and postfault trajectories are obtained from PSS/E computer package developed by Power Technologies (PTI). Several operation points were generated in order to test the FHRCNN method on a stressed system, and in order to study the method's robustness to variations in the operation point. Our base case was obtained by increasing the real powers of the individual load by $25 \%$. The extra power generation was spread uniformly among the generators. We choose an increase of $25 \%$ because it lowered critical clearing times, while maintaining an acceptable load-flow solution. Fifty operating points are generated from base case by considering random changes of key parameters like load, shunt compensation, active and reactive generation scheduling, and topology. The distribution of the random numbers was uniform rather than Gaussian, and a different string of random numbers was used for each operating point. A bus fault refers to a fault on the end of a transmission line which is cleared by removing the line. A mid-line fault refers to a fault in the middle of a transmission line. For the training set, we simulate 650 bus faults and 350 line faults per operation point, in which 330 faults are unstable and 670 are stable. For the test set, we simulate 350 bus faults and 150 line faults, in which 165 faults are unstable and 335 are stable, separate from training set per operating point.

\subsection{Results}

The simulation programs were developed on a SUNSPARC II in $\mathrm{C}^{++}$. For a comparison, we conducted simulation results on traditional feedword neural network $(\mathrm{FNN})$ with the same neurons, training set, and test set. The proposed FHRCNN and FNN were trained on the training set using 61 neurous. It takes about $2 \mathrm{~h}$ to train FHRCNN and about $3 \mathrm{~h}$ to train FNN. Both networks are tested on the test set. It takes just a few hundredths of a second to predict the swings in the first second after clearing time for both networks. The classification rates of both networks are given in Table 1 . The numbers indicate the percentage of predictors correctly classified.

From simulation results, one has the following observations: (1) the FHRCNN has a pretty high classification rate; (2) the FHRCNN has a better performance than traditional FNN; the FHRCNN FHRCNN has the potential to be an on-line tool for real-time transient stability prediction prediction in power systems.

\section{Conclusion}

We have demonstrated the success of properly trained FHRCNN in predicting transient stability from a short window of postfault phasor measurements. Extensive testing was carried out on the IEEE 39-bus system under heavy loading conditions. The computational burden proved to be quite reasonable, and larger systems could be handled. Since individual faults are generated independently, parallel implementation is trivial.

Current financial and environmental trends portend a power system forced to operate under more stressful conditions than in the past. This leaves little room for traditional avoidance principles and will no doubt lead to the use of more control. The transient stability prediction method discussed in this paper is designed to provide timely information for power system control in a real-time. We suggest that a neuro-fuzzy approach can automate the process of transforming off-line simulation studies into on-line decision rules.

\section{References}

[1] J.S. Thorp, A.G. Phadke, S.H. Horowitz, M.M. Begovic, Some applications of phasor measurements to adaptive protection, IEEE Trans. Power Syst. 3 (2) (1988) 791-798.

[2] A.G. Phadke, Synchronized phasor measurements in power systems, IEEE Comput. Appl. Power 6 (2) (1993) 10-15.

[3] A.G. Phadke et al., Synchronized Sampling and Phasor Measurements for Relaying and Control, IEEE PES Winter Meeting, Columbus, OH, 1993 (93 WM 039-8-PWRD).

[4] V. Centeno, et al., Adaptive out-of-step relaying using phasor measurement techniques, IEEE Comput. Appl. Power 6 (4) (1993) $12-17$.

[5] P. Denys, et al., Measurement of voltage phase for the French future defence plan against losses of synchronism, IEEE Trans. PWRD 7 (1992) 62-69.

[6] C.W. Taylor, J.M. Haner, L.A. Hill, W.A. Mittelstadt, R.L. Cresap, A new out-of-step relay with rate of change of apparent resistance augmentation, IEEE Trans. Power Appar. Syst. 102 (3) (1983) 631-639.

[7] H.J. Boenig, J.F. Hauer, Commissioning tests of the Bonueville power administration 30 MJ SMES unit, IEEE Trans. Power Appar. Syst. 104 (2) (1985) 301-312. 
[8] A.G. Phadke, J.S. Thorp, Computer Relaying for Power Systems, Wiley, New York, 1988.

[9] S. Rovnyak, C.-W. Liu, J. Lu, W. Ma, H. Thorp, Predicting future behaviour of transient events rapidly enough to evaluate remedial control options in real-time, IEEE Trans. Power Syst. 10 (3) (1995) 1195-1203.

[10] M.C. Su, A Neural Network Approach to Knowledge Acquisition, Ph.D. Thesis, University of Maryland, MD, 1993.

[11] P.M. Anderson, A.A. Fouad, Power System Control and Stability, Iowa State University Press, Ames, IA, 1977.
[12] M.A. Pai, Energy Function Analysis for Power System Stability, Kluwer, Boston, 1989.

[13] A.A. Fouad, et al., Dynamic security assessment practices in North America, IEEE Trans. Power Syst. 3 (3) (1988) 13101321.

[14] D.J. Sobajic, Y.H. Pao, Artificial neural-net based dynamic security assessment for electric power systems, IEEE Trans. PWRS 4 (1) (1989) 220-228.

[15] C.-W. Liu, J.S. Thorp, Application of synchronized phasor measurements to real-time transient stability prediction, IEE Proc. Gener. Transm. Distrib. 142 (4) (1995) 355-360. 